\title{
Does the routine histological examination of the inferior mesenteric artery lymph nodes have a prognostic value in elderly patients with sigmoid colon and rectum tumors?

\author{
M Fava, A Turoldo*, M Roseano, P Makovac and G Liguori
}

Address: Istituto di Clinica Chirurgica, Università degli Studi di Trieste, Italy

* Corresponding author

from XXI Annual Meeting of The Italian Society of Geriatric Surgery

Terni, Italy. 4-6 December 2008

Published: I April 2009

BMC Geriatrics 2009, 9(Suppl I):A85 doi: 10.1 I86/I47|-23I8-9-SI-A85

This abstract is available from: http://www.biomedcentral.com//47I-23I8/9/SI/A85

(c) 2009 Fava et al; licensee BioMed Central Ltd.

\section{Introduction}

The purpose of our study is to estimate the frequency of the histopathological exam, analyze the prognostic/therapeutic value of central LN examination and determine their advantage in elderly patients.

\section{Materials and methods}

This retrospective study analyzes 519 patients who were hospitalized from 1980 to 2008 at the "Istituto di Clinica Chirurgica" of the University of Trieste and underwent curative surgery for a sigmoid colon and/or rectum tumor (minimum follow up period of 4 years): 311 males and 208 females, mean age of 66.95 (range: 17-94 years). 192 patients were younger than 65,211 were between 65 and 74,109 between 75 and 84 and 7 older than 84 years.

In cases of complete tumor resection and complete lymphadenectomy the surgery was considered as curative. The tumor was located on sigmoid colon in 147 cases, on rectum in 346 cases (high rectum 101, middle rectum 122 and low rectum 123 cases) and in 26 on both.

372 anterior rectal resections, 20 Hartman's resections, 85 Miles resections and 42 segmental colonic resections were performed. The choice of the surgical technique, resection or abdominoperineal amputation, was defined by the tumor location; stage, dimension and biological characteristics were not taken into account.
In all the cases of subperitoneal tumors we performed a total mesorectal excision associated with IMA LN dissection all the way to its origin from the aorta. In 42 cases among patients with poor general conditions and/or high operative risk, with the local state of the tumor taken into account, we performed only a I/II level lymphadenectomy. However, in all the cases, the purpose was to dissect at least $13 \mathrm{LN}$, which should represent the cut-off for a precise staging of the tumor. Those LN are often located in the proximity of the tumor or along the colon wall. That is why, in order to retrieve the LN, we opted for examination of the surgical specimen immediately after the dissection. LN located less than $5 \mathrm{~cm}$ from the tumor were classified as marginal, those along the branches of the mesenteric artery or more than $5 \mathrm{~cm}$ from the tumor as intermediate and those at the origin of the IMA as central. The surgical specimen was then analyzed and the LN are classified by the pathologist as well.

The Kaplan-Meier actuarial method was used to calculate the cause-specific survival rate. The Log-rank test was used to compare the results. The Cox regression was used as a multivariate analysis. The SPSS software was used for the statistical procedures.

\section{Results}

In 306 cases (59.0\%) the LN were retrieved from the surgical specimen and histologically evaluated. In 26 of those $(8.5 \%)$ they were metastatic. The retrieval/identification of the LN was not influenced by the patients' age, with the 
exception of patients older than 84 years. In those patients the histological determination decreased by $14.3 \%$ (64.6\% in the first, $54.5 \%$ in the second, 60.65 in the third, and $14.3 \%$ in the fourth age group, $\mathrm{p}=0.017$ ).

As regards the location of the tumor the central LN identification was possible in: $58.5 \%$ cases of sigmoid colon tumor, $60.4 \%$ of high rectum tumor, 63.95 of middle rectum tumor, $52 \%$ of low rectum tumor and $65.4 \%$ synchronous tumors $(\mathrm{p}=0.317)$.

The mortality and morbidity rate among patients in which the histological evaluation of central LN was possible and those in which it wasn't were not statistically significant (the mortality rate was $3.1 \%$ and $2.9 \%$, the overall morbidity rate was $38 \%$ and $31 \%$, the specific morbidity rate (fistulae) was $6.8 \%$ and $4.9 \%$ respectively).

The actuarial survival rate at 5 years was $73.2 \%$ in patients in which the central $\mathrm{LN}^{\prime}$ status was determinated and $62.7 \%$ in those in which it wasn't $(p=0.0066)$. As regards the stage of the disease the actuarial survival rate was respectively $85.1 \%$ and $82.2 \%$ for Dukes A (patients with central LN' determined status and those in which it was undetermined $),(p=0.5213), 79.2 \%$ and $64.36 \%$ for Dukes $\mathrm{B},(\mathrm{p}=0.0123)$ and $56.1 \%$ and $34.7 \%$ or Dukes C $(\mathrm{p}=0.0468)$ patients.

The actuarial survival rate in patients older than 75 years was $73.4 \%$ in those in which the central LN status was evaluated and $53.57 \%$ in those in which it wasn't ( $\mathrm{p}=$ 0.1122 ). As regards the number of patients the groups were comparable:

Dukes A 14 patients each, Dukes B 44 patients in the first group and 32 in the second, Dukes C 15 patients each (p $=0.719$ ).

Considering the Dukes stage, the "T" of the tumor, the histological evaluation of the central LN and the grading of the tumor as independent variables (results that were statistically significant at the univariate analysis), the Cox logistic regression assigned an independent value only to the Dukes stage [Exp (B) 2,082] and the central lymph node evaluation [Exp (B) 1,402]. The same analysis applied to the elderly patients assigned an independent prognostic value to the Dukes stage only.

\section{Conclusion}

In our experience, in the cases of sigmoid colon and rectum tumors, the histological evaluation of the central LN has a precise prognostic value. Patients in which the histological evaluation of the central LN was performed had a better prognosis than patients in which the histological evaluation wasn't possible, considering patients with tumors in the same stage. In the Dukes A cases the histological evaluation of the central LN didn't have a prognostic/therapeutic value. The histological evaluation of the central LN represents the quality standard of the lymphadenectomy and ensures a precise staging of the tumor, which decreases the shifting between tumor stages. From an oncological point of view the therapeutic improvement achieved can be ascribed to metastatic LN dissection. The advantage of the precise $\mathrm{LN}$ evaluation is confirmed, although not as statistically significant, even in the elderly patients. Therefore, we recommend the dissection/research of the central LN even in this group of patients.

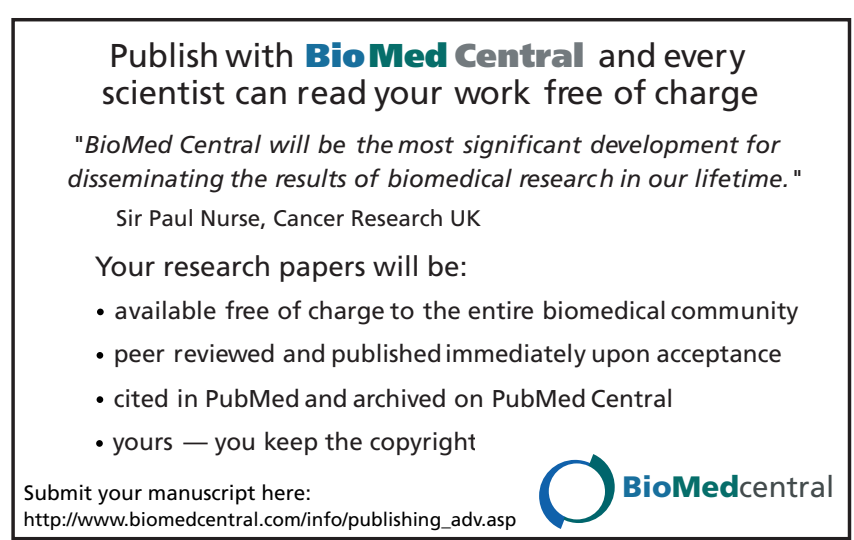

